\title{
Chapter 15 \\ Epidemiological Models Incorporating Mobility, Behavior, and Time Scales
}

\subsection{Introduction}

The work of Eubank et al. [24], Sara del Valle et al. [44], Chowell et al. [7, 18], and Castillo-Chavez and Song [13] have highlighted the impact of modified modeling approaches that incorporate heterogeneous modes of mobility within variable environments in order to study their impact on the dynamics of infectious diseases. Castillo-Chavez and Song [13], for example, proceeded to highlight a Lagrangian perspective, that is, the use of models that keep track at all times of the identity of each individual. This approach was used to study the consequences of deliberate efforts to transmit smallpox in a highly populated city, involving transient subpopulations and the availability of massive modes of public transportation.

Here, a multi-group epidemic Lagrangian framework where mobility and the risk of infection are functions of patch residence time and local environmental risk is introduced. This Lagrangian approach has been used within classical contact epidemiological (that is, transmission is due to "contacts" between individuals) formulations in the context of a possible deliberate release of biological agents $[2,13]$. The Lagrangian approach is introduced here as a modeling approach that explicitly avoids the assignment of heterogeneous contact rates to individuals. The use of contacts or activity levels and the view that transmission is due to collisions between individuals has a long history and it is conceptually consistent with the way we envision disease transmission between susceptible and infectious individuals. However, contacts are hard to define and consequently, at least in the context of communicable diseases, impossible to measure in various settings. Is it possible to capture interactions of individual mathematically in a way different from the notion of contacts? The approach that is proposed focuses on the use of modeling frameworks that involve patches/environments defined or characterized by risks of infection that are functions of the time spent in each environment/patch. These patches/environments may or may not have permanent hosts and they may be used to account for places of "transitory" residence like mass transportation systems or 
hospitals or forests, to name but a few possibilities. Each environment or patch is characterized by the expected risk of infection of visitors as a function of time spent in such an environment. For example, a population near a forest may have some of its individuals spend time in the forest. Those who like the outdoors may be exposed longer to vectors than those who do not visit the forest. Consequently, the possibility of acquiring a vector-borne disease is a function of, among other factors, how long an individual spends in the forest each day. Similarly, individuals that use mass transportation routinely (during rush hour) are at a higher risk of acquiring a communicable disease including common colds, and it makes sense to assume that the risk may be a function of how long each individual spends each day commuting to work or to school. In other words, the average time spent in a community defined as a collection of environments that determined a priori the risk of acquiring an infection is at the heart of the Lagrangian approach.

What is the Lagrangian approach and what does the theory tell us about the dynamics of such models in epidemic settings? We revisit this framework in possibly the simplest general setting that of a susceptible-infected-susceptible (SIS) epidemic multi-group model. We collect some of the mathematical formulae and results in the context of this general SIS multi-group model as reported in the literature $[4,6,7,11]$. We proceed to identify basic reproduction numbers $\mathscr{R}_{0}$ as a function of the associated multi-patch residence-time matrix $\mathbb{P}\left(p_{i, j}: i, j=\right.$ $1,2,3 \ldots n)$, which determines the proportion of time that a resident of Patch $i$ spends in environment $j$. The analysis shows that the $n$-patch SIS model (as long as it is a strongly connected system) has a unique globally stable endemic equilibrium when $\mathscr{R}_{0}>1$, and a globally stable disease-free equilibrium when $\mathscr{R}_{0} \leq 1$. We have used simulations to generate insights on the impact that the residence matrix $\mathbb{P}$ has on infection levels within each patch. Model results $[4,6,7,11]$ show that the infection risk vector, which characterizes environments by risk to a pre-specified disease (measured by $\mathbb{B}$ ), and the residence-time matrix $\mathbb{P}$ both play an important role in determining, for example, whether or not endemicity is reached at the patch level. Further, it is shown that the right combinations of environmental risks $(\mathbb{B})$ and mobility behavior $(\mathbb{P})$ are capable of promoting or suppressing infection within particular patches. The theoretical results $[4,6,7,11]$ are used to characterize patchspecific disease dynamics as a function of the time spent by residents and visitors in patches of interest. These results have helped classify patches as sources or sinks of infection, depending, of course, on the risk $(\mathbb{B})$ and mobility $(\mathbb{P})$ matrices. In general a residence-time matrix $\mathbb{P}$ cannot be made of constant entries in realistic settings. In fact the entries of $\mathbb{P}$ may depend on disease prevalence levels. We have explored some simple situations, via simulations, when the entries of the matrix $\mathbb{P}$ are state-dependent $[4,6,7,11]$. The analysis and simulations for specific diseases are illustrated later in this chapter. They are used to highlight some of the possible differences that arise from having a state-dependent residence-time matrix $\mathbb{P}$. 


\subsection{General Lagrangian Epidemic Model in an SIS Setting}

The following SIS model involving $n$-patches (environments) is introduced in [7]:

$$
\begin{aligned}
S_{i}^{\prime} & =b_{i}-d_{i} S_{i}+\gamma_{i} I_{i}-S_{i} \lambda_{i}(t) \\
I_{i}^{\prime} & =S_{i} \lambda_{i}(t)-\gamma_{i} I_{i}-d_{i} I_{i} \\
N_{i}^{\prime} & =b_{i}-d_{i} N_{i},
\end{aligned}
$$

where $b_{i}, d_{i}$, and $\gamma_{i}$ denote the per-capita birth, natural death, and recovery rates, respectively, for $i=1,2,3 \ldots n$. The infection rates $\lambda_{i}(t)$ have the form:

$$
\lambda_{i}(t)=\sum_{j=1}^{n} \beta_{j} p_{i j} \frac{\sum_{k=1}^{n} p_{k j} I_{k}}{\sum_{k=1}^{n} p_{k j} N_{k}}, \quad i=1,2, \ldots, n,
$$

where $p_{i j}$ denotes the proportion of susceptibles from Patch $i$ who are currently in Patch $j, \beta_{j}$ is the risk of infection in Patch $j$, and the last fraction represents the proportion of infected in Patch $j$. Using the approach of the next generation matrix, the basic reproduction number $\mathscr{R}_{0}$ can be derived using the following system:

$$
\dot{I}_{i}=\left(\frac{b_{i}}{d_{i}}-I_{i}\right) \lambda_{i}(t)-\left(\gamma_{i}+d_{i}\right) I_{i}, \quad i=1,2, \ldots, n .
$$

As shown in the next section, $\mathscr{R}_{0}$ is a function of the risk vector $\mathscr{B}=$ $\left(\beta_{1}, \beta_{2}, \ldots, \beta_{n}\right)^{t}$ and the residence times matrix $\mathbb{P}=\left(p_{i j}\right), i, j=1, \ldots, n$, and it is shown in [7] that whenever $\mathbb{P}$ is irreducible (patches are strongly connected), the disease-free steady state is globally asymptotically stable if $\mathscr{R}_{0} \leq 1$ and a unique interior equilibrium exists and is globally asymptotically stable if $\mathscr{R}_{0}>1$.

While a specific formula for the multi-patch basic reproduction number cannot be computed explicitly, it is possible in this case to find expressions for the patchspecific basic reproduction number. In fact, we have

$$
\mathscr{R}_{0 i}(\mathbb{P})=\mathscr{R}_{0 i} \times \sum_{j=1}^{n} p_{j i}
$$

where $\mathscr{R}_{0 i}$ are the local basic reproduction numbers $(i=1,2,3, \cdots, n)$ computed when the patches are isolated from each other. From the $\mathscr{R}_{0 i}(i=1,2,3, \cdots, n)$, the role that the relative risk that each environment (patch) plays, namely $\frac{\beta_{j}}{\beta_{i}}$, can be assessed. Further, the role that residence times play in keeping track of the appropriate fraction of the population involved in a given patch is given by $\frac{\left(p_{i j} b_{i} / d_{i}\right)}{\sum_{k=1}^{n} p_{k j} b_{k} / d_{k}}$. In other words, this patch specific $\mathscr{R}_{0 i}(i=1,2,3, \cdots$,$) captures$ the impact of the $\mathbb{P}$ and $\mathbb{B}$ matrices. 
In short, if $\mathscr{R}_{0 i}(\mathbb{P})>1$ the disease persists in Patch $i$ and furthermore, if $p_{k j}=0$ for all $k=1,2, \cdots, n$ and $k \neq i$ whenever $p_{i j>1}$, then it is shown that the disease dies in Patch $i$ if $\mathscr{R}_{0 i}(\mathbb{P})<1$, that is, patch-specific basic reproduction numbers help characterize disease dynamics at the patch level [7].

We can look first at the following example of a multi-patch SIR model for a single outbreak:

$$
\begin{aligned}
& S_{i}^{\prime}=-S_{i} \lambda_{i}(t), \\
& I_{i}^{\prime}=S_{i} \lambda_{i}(t)-\alpha_{i} I_{i}, \\
& R_{i}^{\prime}=\alpha_{i} I_{i}, \quad i=1,2,
\end{aligned}
$$

where $S_{i}, I_{i}$, and $R_{i}$ denote the population of susceptible, infected, and recovered immune individuals, respectively, in Patch $i$, and $N_{i}=S_{i}+I_{i}+R_{i}$. This model is the same as the model (14.7-14.8) in the preceding chapter. The parameter $\alpha_{i}$ denotes the per-capita recovery rate in Patch $i$ and $\lambda_{i}(t)$ are given in (15.2).

In the rest of this chapter we make use of this Lagrangian modeling perspective (disease-specific versions) to carry out preliminary studies, in rather simple set ups, of the role of mobility in reducing or enhancing the transmission of specific diseases in regions of variable risk for the case of two patches. Numerical results are used to illustrate the power and limitations of this approach. Lagrangian models are used to explore the role that mobility plays in disease transmission for the cases of Ebola, tuberculosis, and Zika in simplified settings. Figure 15.1 represents a schematic representation of the Lagrangian dispersal between two patches.

\section{Patch 1}
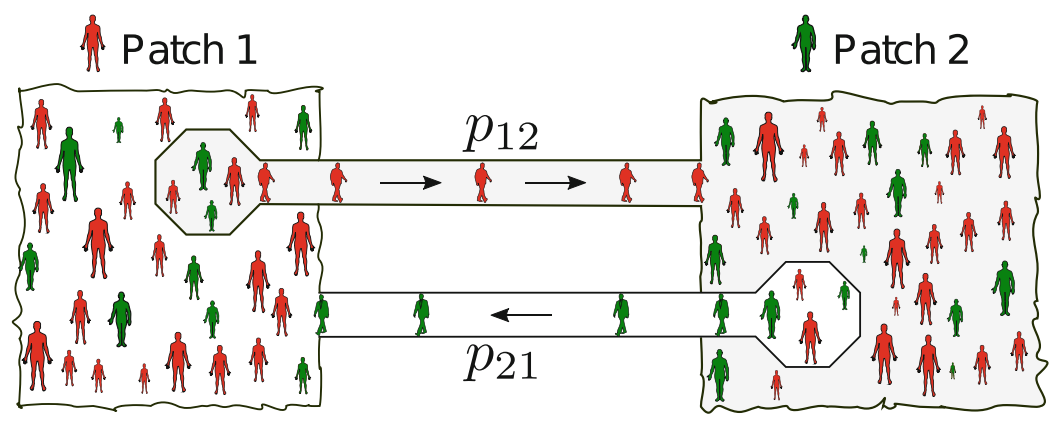

Fig. 15.1 Dispersal of individuals via a Lagrangian approach 


\subsection{Assessing the Efficiency of Cordon Sanitaire as a Control Strategy of Ebola}

During the 2014 Ebola Epidemic in West Africa, it was observed [10, 46] that the rate of growth of the Ebola epidemic seemed to be increasing rather than decreasing as is standard in the study of epidemics. In other words, the reproduction number tends to decrease in time rather than increase. The evidence provided by the data and our analysis indicated that something was not right. We learned that troops were being used to prevent individuals from moving out of the most devastated communities facing Ebola. The use of cordons sanitaires seemed to be implemented even though past experiences have shown them to have a deleterious effect. Here, we formulate a two-patch mathematical model for Ebola virus disease (EVD) dynamics to highlight the potential lack of effectiveness or the deleterious impact of impeding mobility (cordons sanitaires). Via simulations, we look at the role of mandatory mobility restrictions and their impact on disease dynamics and epidemic final size. It is shown that mobility restrictions between high and low risk areas of closely linked communities are likely to have a deleterious impact on overall levels of infection in the total population involved.

\subsubsection{Formulation of the Model}

The community of interest is assumed to be composed of two adjacent regions facing highly distinct levels of EVD infection and having access to a highly differentiated public health system (the haves and have-nots). There are differences in population density, availability of medical services, and isolation facilities. The need of those in the high-risk area to travel to the low-risk area is high as the jobs are in the well-off community. For Ebola, it may be unrealistic to assume susceptibles and infectives travel at the same rate. We let $N_{1}$ denote the population in Patch 1 (high risk) and $N_{2}$ the population in Patch 2 (low risk). The classes $S_{i}, E_{i}, I_{i}, R_{i}$ represent the susceptible, exposed, infective, and recovered sub-populations in Patch $i(i=1,2)$. The class $D_{i}$ represents the number of disease induced deaths in Patch $i$. The dispersal of individuals is modeled via the Lagrangian approach defined in terms of residence times $[4,7]$.

The numbers of new infections per unit of time are based on the following assumptions:

- The density of infected individuals mingling in Patch 1 at time $t$, who are only capable of infecting susceptible individuals currently in Patch 1 at time $t$, that is, the effective infectious proportion in Patch 1 is given by

$$
\frac{p_{11} I_{1}+p_{21} I_{2}}{p_{11} N_{1}+p_{21} N_{2}},
$$


where $p_{11}$ denotes the proportion of time that residents from Patch 1 spend in Patch 1 and $p_{21}$ the proportion of time that residents from Patch 2 spend in Patch 1 .

- The number of newly infected Patch 1 residents while sojourning in Patch 1 is therefore given by

$$
\beta_{1} p_{11} S_{1}\left(\frac{p_{11} I_{1}+p_{21} I_{2}}{p_{11} N_{1}+p_{21} N_{2}}\right) .
$$

- The number of new infections within members of Patch 1, in Patch 2 per unit of time is therefore

$$
\beta_{2} p_{12} S_{1}\left(\frac{p_{12} I_{1}+p_{22} I_{2}}{p_{12} N_{1}+p_{22} N_{2}}\right),
$$

where $p_{12}$ denotes the proportion of time that residents from Patch 1 spend in Patch 2 and $p_{22}$ the proportion of time that residents from Patch 2 spend in Patch 2. Hence, the effective density of infected individuals in Patch $j$ is given by

$$
p_{1 j} N_{1}+p_{2 j} N_{2}, \quad j=1,2 .
$$

If we further assume that infection by dead bodies occurs only at the local level (bodies are not moved), then, by following the same rationale as in model (15.3), we arrive at the following model:

$$
\begin{aligned}
& S_{i}^{\prime}=-S_{i} \lambda_{i}(t)-\varepsilon_{i} \beta_{i} p_{i i} S_{i} \frac{D_{i}}{N_{i}}, \\
& E_{i}^{\prime}=S_{i} \lambda_{i}(t)+\varepsilon_{i} \beta_{i} p_{i i} S_{i} \frac{D_{i}}{N_{i}}-\kappa E_{i}, \\
& I_{i}^{\prime}=\kappa E_{i}-\gamma I_{i}, \\
& D_{i}^{\prime}=f_{\mathrm{d}} \gamma I_{i}-\nu D_{i}, \\
& R_{i}^{\prime}=\left(1-f_{\mathrm{d}}\right) \gamma I_{i}+\nu D_{i}, \\
& N_{i}=S_{i}+E_{i}+I_{i}+D_{i}+R_{i}, \quad i=1,2,
\end{aligned}
$$

where $\lambda_{i}(t)$ are given in (15.2).

\subsubsection{Simulations}

Simulations show that if only individuals from the high-risk region (Patch 1) were allowed to travel, then the epidemic final size can go under the cordon sanitaire level. Figure 15.2 captures the patch-specific prevalence levels for mobility values of $p_{12}=0,0.2,0.4,0.6$ with $p_{21}=0$ (no movement). Disease dispersal, if the 

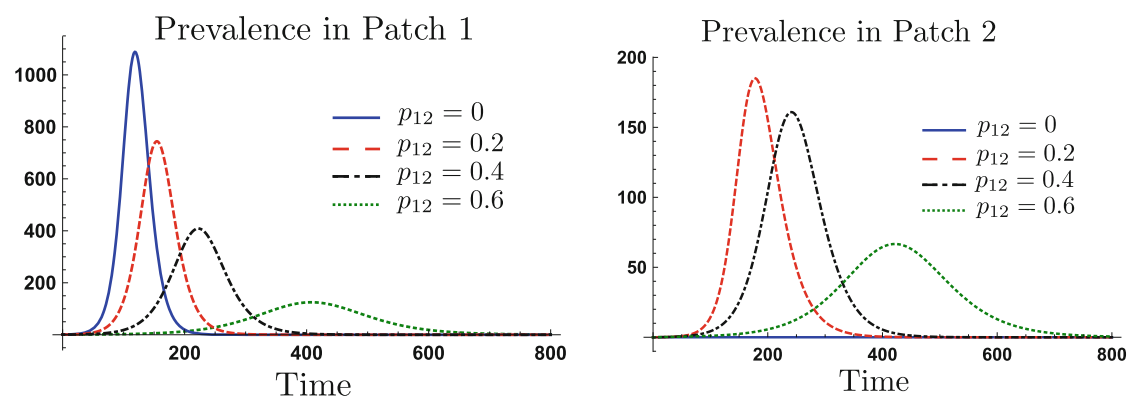

Fig. 15.2 Dynamics of prevalence in each patch for values of mobility $p_{12}=$ $0 \%, 20 \%, 40 \%, 60 \%$ and $p_{21}=0$, with parameters: $\varepsilon_{1,2}=1.1, \mathscr{R}_{01}=2.45, \mathscr{R}_{02}=$ $0.9, f_{\mathrm{d}}=0.7, k=1 / 7, v=1 / 2, \gamma=1 / 7$

Final epidemic size Patch 2


Fig. 15.3 Dynamics of patch specific and total final epidemic size, for mobility values $p_{12}=$ $0 \%, 20 \%, 40 \%, 60 \%$ and $p_{21}=0$, with parameters: $\varepsilon_{1,2}=1.1, \mathscr{R}_{01}=2.45, \mathscr{R}_{02}=0.9, f_{\mathrm{d}}=$ $0.7, k=1 / 7, v=1 / 2, \gamma=1 / 7$

disease spreads to a totally susceptible region, means that the secondary infections produced in the low-risk region reduce the overall two-patch prevalence, due to its access to better sanitary conditions and resources. However, there is a cost to the low-risk patch not only for the services provided but also for the generation of a larger number of secondary cases than if the "borders" were closed. Figure 15.3 shows that different mobility regimes can increase or decrease the total epidemic final size. In the presence of "low mobility" levels $\left(p_{12}=0.2,0.4\right)$, the total final size curve may turn out to be greater than the cordon sanitaire case. We observe that the nonlinear impact of mobility on the total epidemic final size can bring it below the cordoned case even under relative "high mobility" regimes. This result highlights the trade-off that comes from reducing individuals' time spent in a highrisk region versus exposing a totally susceptible population living in a safer region. Under certain mobility conditions, the results of such a trade-off are beneficial for the Global Commons. 
Fig. 15.4 Dynamics of maximum final size and maximum prevalence in Patch 1 with parameters: $\varepsilon_{1,2}=$ $1.1, \mathscr{R}_{01}=2.45, \mathscr{R}_{02}=$ $0.9, f_{d}=0.7, k=1 / 7, v=$ $1 / 2, \gamma=1 / 7$

Fig. 15.5 Dynamics of final epidemic size in the one way case with parameters: $\varepsilon_{1,2}=$ $1.1, \mathscr{R}_{01}=2.45, \mathscr{R}_{02}=$ $0.9,1.0,1.1, f_{\mathrm{d}}=0.7, k=$ $1 / 7, v=1 / 2, \gamma=1 / 7$
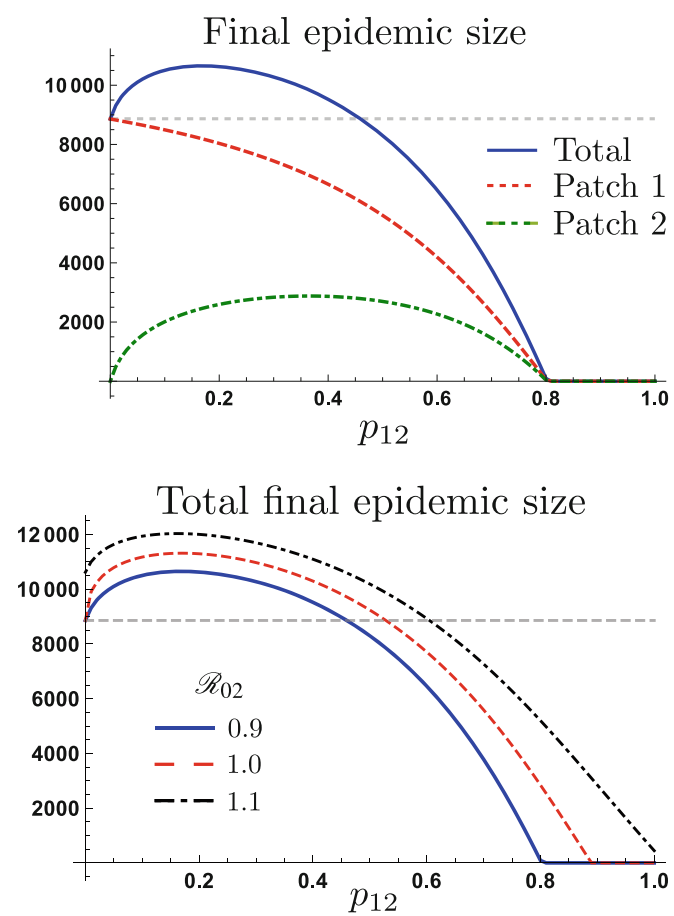

Further, in order to clarify the effects of residence times on total final epidemic size, we proceeded to analyze its behavior under one way mobility. Figure 15.4 shows the cordon sanitaire (dashed gray line), patch specific, and the total epidemic final size for various possible mobility scenarios, $p_{12} \in[0,1]$. We see that one way mobility reduces Patch 1 epidemic final size while increasing the Patch 2 final number of infections. We see that the total epidemic final size under low mobility $\left(p_{12}<0.5\right)$ is above the cordoned case. We also observe that Patch 2 sanitary conditions play an important role under high mobility regime bringing the total epidemic final size below the cordon sanitaire scenario $\left(p_{12}>0.5\right)$.

Moreover, results suggest that for $\mathscr{R}_{02}<1$ extremely high mobility levels might eradicate an Ebola outbreak. It is important to stress that mobility reducing the total epidemic final size is dependent not only on the residence times and mobility type, but also on the patch-specific prevailing infection rates. Figure 15.5 shows that if $\mathscr{R}_{02}>1$ mobility is not capable of leading the total epidemic final size towards zero. Figure 15.6 shows that the global basic reproduction number decreases monotonically as one way mobility increases. However, it is not capable of capturing the harmful effect of low mobility levels, increasing the total epidemic final size. Mobility on its own is not always enough to reduce $\mathscr{R}_{0}$ below the critical 
Fig. 15.6 Dynamics of $\mathscr{R}_{0}$ with parameters:

$\varepsilon_{1,2}=1, \mathscr{R}_{01}=2.45, \mathscr{R}_{02}=$ $0.9,1.0,1.1, f_{\mathrm{d}}=0.708, k=$ $1 / 7, \alpha=0, v=1 / 2, \gamma=$ $1 / 6.5$

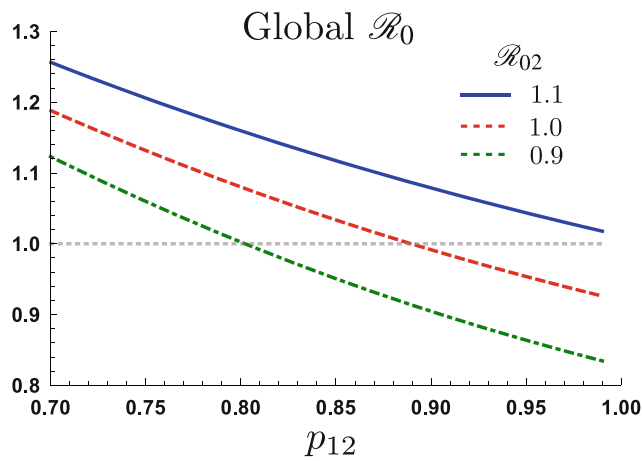

threshold. Instead, bringing the global $\mathscr{R}_{0}$ less than one requires reducing local risk, that is, getting a lower $\mathscr{R}_{02}$.

\section{4 *Mobility and Health Disparities on the Transmission Dynamics of Tuberculosis}

TB dynamics is the result of complex epidemiological and socio-economical interactions between and among individuals living in highly heterogeneous regional conditions. Many factors impact TB transmission and progression. A model is introduced to enhance the understanding of TB dynamics in the presence of diametrically distinct rates of infection and mobility. The dynamics are studied in a simplified world consisting of two patches, that is, two risk-defined environments, where the impact of short-term mobility and variations in reinfection and infection rates are assessed. The modeling framework captures "daily dynamics" of individuals within and between places of residency, work, or business. Activities are modeled by the proportion of time spent in environments (patches) having different TB infection risk. Mobility affects the effective population size of each Patch $i$ (home of $i$ residents) at time $t$ and they must also account for visitors and residents of Patch $i$, at time $t$. The impact that effective population size and the distribution of individuals' residence times in different patches have on TB transmission and control is explored using selected scenarios where risk is defined by the estimated or perceived first time infection and/or exogenous reinfection rates. Model simulation results suggest that, under certain conditions, allowing infected individuals to move from high to low TB prevalence areas (for example, via the sharing of treatment and isolation facilities) may lead to a reduction in the total TB prevalence in an overall, twopatch, population. 


\subsection{1 *A Two-Patch TB Model with Heterogeneity in Population Through Residence Times in the Patches}

Using a similar approach to model formulation, we consider the following model for the dynamics of TB in two patches:

$$
\begin{aligned}
& \dot{S}_{i}=\mu_{i} N_{i}-S_{i} \lambda_{i}(t)-\mu_{i} S_{i}, \\
& \dot{L}_{i}=q S_{i} \lambda_{i}(t)-L_{i} \hat{\lambda}_{i}(t)-\left(\gamma_{i}+\mu_{i}\right) L_{i}+\rho_{i} I_{i}, \\
& \dot{I}_{i}=(1-q) S_{i} \lambda_{i}(t)+L_{i} \hat{\lambda}_{i}(t)+\gamma L_{i}-\left(\mu_{i}+\rho_{i}\right) I_{i}, \quad i=1,2,
\end{aligned}
$$

where $\lambda_{i}(t)$ is the same as in (15.2) and

$$
\hat{\lambda}_{i}(t)=\sum_{j=1}^{2} \delta_{j} p_{i j} \frac{\sum_{k=1}^{2} p_{k j} I_{k}}{\sum_{k=1}^{2} p_{k j} N_{k}}, \quad i=1,2 .
$$

\subsection{2 *Results: The Role of Risk and Mobility on TB Prevalence}

We highlighted the dynamics of tuberculosis within a two-patch system, described by (15.5), under various residence times schemes via numerical experiments. The simulations were carried out using the two-patch Lagrangian modeling framework on pre-constructed scenarios. We assume that one of the two regions (say, Patch 1) has high TB prevalence. We do not model specific cities or regions. Nomenclature of some terms and scenarios are defined in Table 15.1.

The interconnection of the two idealized patches demands that individuals from Patch 1 travel to the "safer" Patch 2 to work, to school, or for other social activities. It is assumed that the proportion of time that Patch 2 residents spend in Patch 1 is negligible.

Here we define "high risk" based on the value of the probability of developing active TB using two distinct definitions: (i) patch having high direct first time transmission potential but no difference in exogenous reinfection potential between patches $\left(\beta_{1}>\beta_{2}\right.$ and $\left.\delta_{1}=\delta_{2}\right)$ and (ii) the patch with high exogenous reinfection potential $\left(\delta_{1}>\delta_{2}\right.$ and $\left.\beta_{1}=\beta_{2}\right)$. In addition, we assume a fixed population size for Patch 1 and vary the population size of Patch 2. Particularly, we assume that Patch 1 is the denser patch, while Patch 2 is assumed to have $\frac{1}{2} N_{1}$ and $\frac{1}{4} N_{1}$. That is, contact rates are higher in the Patch 1 population as compared to corresponding rates in Patch 2. 
Table 15.1 Definitions and scenarios

\begin{tabular}{l|l}
\hline Nomenclature & $\begin{array}{l}\text { Interpreted based on levels of infection rate, prevalence, or } \\
\text { average contacts (via population size) }\end{array}$ \\
\hline Risk & $\begin{array}{l}\text { Defined either by high direct first time infection rate (i.e., high } \beta \\
\text { which leads to high corresponding } \mathscr{R}_{0} \text { ) or by high exogenous } \\
\text { reinfection rate (i.e., high } \delta \text { ) }\end{array}$ \\
\hline $\begin{array}{l}\text { Enhanced } \\
\text { socio-economic } \\
\text { conditions (reducing } \\
\text { health disparity) }\end{array}$ & $\begin{array}{l}\text { Defined by better healthcare infrastructure which is incorporated } \\
\text { by high prevalence of a disease (i.e., high } I(0) / N \text { ) in a large } \\
\text { population (i.e., large } N \text { ) }\end{array}$ \\
\hline Mobility & $\begin{array}{l}\text { Captured by average residence times of an individual in different } \\
\text { patches (i.e., by using } \mathbb{P} \text { matrix) }\end{array}$ \\
\hline
\end{tabular}

Scenarios (assume high-risk and diminished socio-economic conditions in

Patch 1 as compared to Patch 2)

\begin{tabular}{l|l}
\hline Scenario 1 & $\begin{array}{l}\beta_{1}>\beta_{2}, \delta_{1}=\delta_{2} ; \frac{I_{1}(0)}{N_{1}}>\frac{I_{2}(0)}{N_{2}}, N_{1}>N_{2} ; \\
\text { vary } p_{12} \& p_{21} \approx 0\end{array}$ \\
\hline Scenario 2 & $\begin{array}{l}\beta_{1}=\beta_{2}, \delta_{1}>\delta_{2} ; \frac{I_{1}(0)}{N_{1}}>\frac{I_{2}(0)}{N_{2}}, N_{1}>N_{2} ; \\
\text { vary } p_{12} \& p_{21} \approx 0\end{array}$ \\
\hline
\end{tabular}
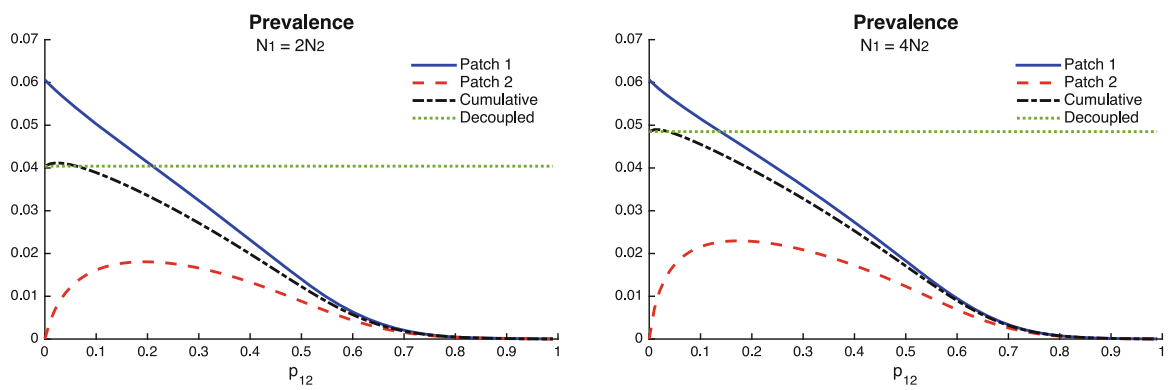

Fig. 15.7 Effect of mobility in the case of different transmission rates $0.13=\beta_{1}>\beta_{2}=0.07$ (which gives $\mathscr{R}_{01}=1.5, \mathscr{R}_{02}=0.8$ ) and $\delta_{1}=\delta_{2}=0.0026$, on the endemic prevalence. The cumulative prevalence and prevalence for each patch using the following population size proportions $N_{2}=\frac{1}{2} N_{1}$ (left figure) and $N_{2}=\frac{1}{4} N_{1}$ (right figure) are shown here. The green horizontal dotted line represents the decoupled case (i.e., the case when there is no movement between patches)

\subsubsection{The Role of Risk as Defined by Direct First Time Transmission Rates}

In this subsection, we explore the impact of differences in transmission rates between patches. Patch 1 is high risk $\left(\mathscr{R}_{01}>1\right.$; obtained by assuming $\left.\beta_{1}>\beta_{2}\right)$, while Patch 2 in the absence of visitors would be unable to sustain an epidemic $\left(\mathscr{R}_{02}<1\right)$. In addition the effect of different population ratios $N_{1} / N_{2}$ is explored. 
Figure 15.7 uses mobility values $p_{12}$ as it looks at their impact on increases in cumulative two-patch prevalence. At the individual patch level, increase in mobility values reduces the prevalence in Patch 1 but increases the prevalence in Patch 2 initially and then decreases past a threshold value of $p_{12}$ (see red and black curves in Fig. 15.7). That is, completely cordoning off infected regions may not be a good idea to control disease. However, the movement rate of individuals between highrisk infection region and low-risk region must be maintained above a critical value to control an outbreak. Thus, it is possible that when Patch 1 (riskier patch) has a bigger population size, then mobility may turn out to be beneficial; the higher the ratio in population sizes, the higher the range of beneficial "traveling" times.

\subsubsection{The Impact of Risk as Defined by Exogenous Reinfection Rates}

Here, we focus our attention on the impact of exogenous reinfection on TB's transmission dynamics when transmission rates are the same in both patches, $\beta_{1}=\beta_{2}$. In this scenario, we assume the disease in both patches have reached an endemic state, that is, $\mathscr{R}_{01}>1$ and $\mathscr{R}_{02}>1$. However, Patch 1 remains the riskier, due to the assumption that exogenous reactivation of TB in Patch 1 is higher than in Patch 2, $\delta_{1}>\delta_{2}$.

Figure 15.8 shows the combined role of exogenous reinfection and mobility values when the population of Patch 1 is twice or four times the population of Patch 2.

It is possible to see a small reduction in the overall prevalence, given for all mobility values from Patch 1 to Patch 2 . Within this framework, parameters, and scenarios, our model suggests that direct first time transmission plays a central role
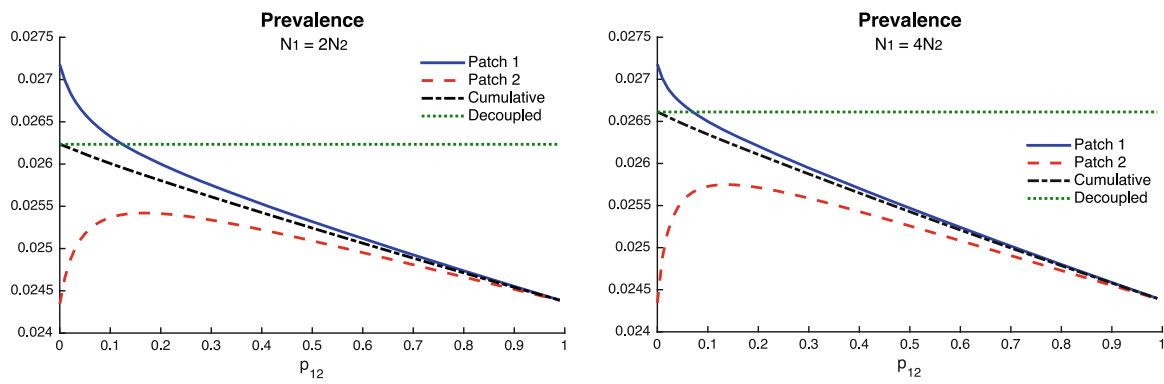

Fig. 15.8 Effect of mobility when risk is defined by the exogenous reinfection rates $0.0053=$ $\delta_{1}>\delta_{2}=0.0026$ and $\beta_{1}=\beta_{2}=0.1$ (which gives $\mathscr{R}_{01}=\mathscr{R}_{02}=1.155$ ), on the endemic prevalence. The cumulative prevalence and prevalence for each patch using the following population size proportions $N_{2}=\frac{1}{2} N_{1}$ (left figure) and $N_{2}=\frac{1}{4} N_{1}$ (right figure) are shown here. The green dotted line represents the decoupled case (i.e., the case when there is no movement between patches) 
Fig. 15.9 Effect of mobility and population size proportions on the global basic reproduction number $\mathscr{R}_{0}$ when

$0.13=\beta_{1}>\beta_{2}=0.07$ and $\delta_{1}=\delta_{2}=0.0026$

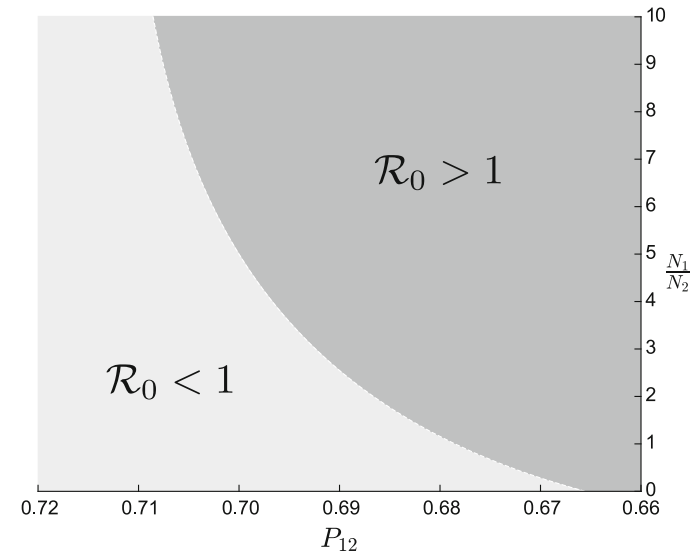

in TB dynamics when mobility is considered. Although mobility also reduces the overall prevalence when exogenous reinfection differs between patches, its impact is small compared to direct first time transmission results.

Finally, Fig. 15.9 shows the relationship between population densities and mobility $\left(p_{12}\right)$ with respect to the basic reproduction number $\mathscr{R}_{0}$. In this case we only explore the first case: direct first time transmission heterogeneity and found out that in this case mobility could indeed eliminate a TB outbreak.

According to the World Health Organization (WHO) [48], in 2014, 80\% of the reported TB cases occurred in 22 countries, all developing countries. Efforts to control TB have been successful in many regions of the globe and yet we still see 1.5 million people die each year. And so, TB, faithful to its history [19], still poses one of the greatest challenges to global health. Recent reports suggest that established control measures for TB have not been adequately implemented, particularly in subSaharan countries $[1,15]$. In Brazil rates have decreased with relapse being more important than reinfection [20,33]. Finally, in Cape Town, South Africa, a study [47] showed that in high incidence areas, individuals who have received TB treatment and are no longer infectious are at the highest risk of developing TB instead of being the most protected. Hence, policies that do not account for population specific factors are unlikely to be effective. Without a complete description of the attributes of the community in question, it is almost impossible to implement successful intervention programs capable of generating low reinfection rates through multiple pathways and low number of drug resistant cases. Intervention must account for the risks that are inherent with high levels of migration as well as with local and regional mobility patterns between areas defined by high differences in TB risk. This discussion of TB dynamics within a simplified framework of a two-patch system has captured in a rather dramatic way the dynamics in two worlds: the world of the haves and the world of the have-nots. Simulations of simplified extreme scenarios highlight the impact of disparities.

TB dynamics depend on the basic reproduction number $\left(\mathscr{R}_{0}\right)$, a function of model parameters that includes direct first transmission and exogenous (reinfection) 
transmission rates. The simulations of specific extreme scenarios suggest that shortterm mobility between heterogeneous patches does not always contribute to overall increases in TB prevalence. The results show that when risk is considered only in terms of exogenous reinfection, global TB prevalence remains almost unchanged when compared to the effect of direct new infection transmission. In the case of a high-risk direct first time transmission, it is observed that mobile populations may contribute to prevalence levels in both environments (patches). The simulations show that when the individuals from the risky population spend $25 \%$ of their time or less in the safer patch this is bad for the overall prevalence. However, if they spend more, the overall prevalence decreases. Further, in the absence of exogenous reinfections, the model is robust, that is, the disease dies out or persists based on whether or not the basic $\mathscr{R}_{0}$ is below or above unity, respectively. Although, the role of exogenous reinfection seems not that relevant to overall prevalence, the fact remains that such mode of transmission increases the risk that comes from a large displacement of individuals into a particular TB-free areas, due to catastrophes or conflict. As noted in [25], ignoring exogenous reinfections, that is, establishing policies that focus exclusively on the reproduction number $\mathscr{R}_{0}$, would amount to ignoring the role of dramatic changes in initial conditions, now more common than before, due to the displacement of large groups of individuals, the result of catastrophes, and/or conflict.

\section{$15.5 *$ ZIKA}

In November 2015, El Salvador reported their first case of Zika virus (ZIKV), an event followed by an explosive outbreak that generated over 6000 suspected cases in a period of 2 months. National agencies began implementing control measures that included vector control and recommending an increased use of repellents. In addition in response to the alarming and growing number of microcephaly cases in Brazil, the importance of avoiding pregnancies for 2 years was stressed. The role of mobility within communities characterized by extreme poverty, crime, and violence where public health services are not functioning is the set up for this example. We use a Lagrangian modeling approach within a two-patch setting in order to highlight the possible effects that short-term mobility, within two highly distinct environments, may have on the dynamics of ZIKV when the overall goal is to reduce the number of cases in both patches. The results of simulations in highly polarized and simplified scenarios are used to highlight the role of mobility on ZIKV dynamics. We found that matching observed patterns of ZIKV outbreaks was not possible without incorporating increasing levels of heterogeneity (more patches). A lack of attention to the threats posed by the weakest links in the global spread of diseases poses a serious threat to global health policies (see [12, 16, 23, 34, 40$42,50])$. Our results highlight the importance of focusing on key nodes of global transmission networks, which in the case of many regions correspond to places where the level of violence is highest. Latin America and the Caribbean, which 
house $9 \%$ of the global population are a particular hot spot because this region accounts for $33 \%$ of the world's homicides [29]. Hence, it is essential to assess how much public safety conditions may affect mobility and the level of local risk, which may affect the dynamics of ZIKV.

\subsection{1 *Single Patch Model}

Assume that individuals while in Patch 1 will be experiencing high risk of infection, while those in Patch 2 will be experiencing low risk. Movement (daily activities) will alter the amount of time that each individual spends on each patch, the longer that an individual is found in Patch 1, the more likely that he/she will become infected. The level of patch-specific risk to infection is captured via the use of a single parameter $\hat{\beta}_{i}, i=1,2$ with $\hat{\beta}_{1} \gg \hat{\beta}_{2}$. This assumption pretends to capture health disparities in a rather simplistic way. The case of Johannesburg and Soweto in South Africa, or North and South Bogota in Colombia, or Rio de Janeiro and adjacent favelas in Brazil, or gang-controlled and gang-free areas within San Salvador are but some of the unfortunately large number of pockets dominated by conflict, high crime or highly differentiated health structures within urban centers around world. The short time scale dynamics of individuals (going to work or attending schools or universities) are incorporated within this model. The dynamics of transmission is carried out via simulations over the duration of a single outbreak.

The ZIKV dynamics single patch model involves host and vector populations of size $N_{h}$ and $N_{v}$, respectively. Both populations are subdivided by epidemiological states; the transmission process is modeled as the result of the interactions of these populations. On that account, we let $S_{h}, E_{h}, I_{h, a}, I_{h, s}$, and $R_{h}$ denote the susceptible, latent, infectious asymptomatic, infectious symptomatic, and recovered host sub-populations. Similarly, $S_{v}, E_{v}$, and $I_{v}$ are used to denote the susceptible, latent, and infectious mosquito sub-populations. Since the focus is on the study of disease dynamics over a single outbreak, we neglect the host demographics while assuming that the vector demographics do not change, meaning that it is assumed the birth and death per-capita mosquito rates cancel each other out. New reports $[14,21]$ point out the presence of large numbers of asymptomatic ZIKV infectious individuals. Consequently, we consider two classes of infectious $I_{h, a}$ and $I_{h, s}$, that is, asymptomatic and symptomatic infectious individuals. Further, since there is no full knowledge of the dynamics of ZIKV transmission, it is assumed that $I_{h, a}$ and $I_{h, s}$ individuals are equally infectious with their periods of infectiousness roughly the same. Our assumptions could be used to reduce the model to one that considers a single infectious class $I_{h}=I_{h, a}+I_{h, s}$. We keep both infectious classes as it may be desirable to keep track of each type. These assumptions may not be too bad given our current knowledge of ZIKV epidemiology and the fact that ZIKV infections, in general, are not severe. Furthermore, given that the infectious process of ZIKV is somewhat similar to that of dengue, we use some of the parameters estimated in dengue transmission studies within El Salvador. ZIKV basic reproduction number 
estimates are taken from those that we just estimated using outbreak data from Barranquilla Colombia [45]. Furthermore, the selection of model parameters ranges used also benefited from prior estimates conducted with data from the 2013-2014 French Polynesia outbreak [31], some of the best available. The dynamics of the prototypic single patch system, single epidemic outbreak, can therefore be modeled using the following standard nonlinear system of differential equations [9]:

$$
\begin{aligned}
& S_{h}^{\prime}=-b \beta_{v h} S_{h} \frac{I_{v}}{N_{h}} \\
& E_{h}^{\prime}=b \beta_{v h} S_{h} \frac{I_{v}}{N_{h}}-v_{h} E_{h} \\
& I_{h, s}^{\prime}=(1-q) v_{h} E_{h}-\gamma_{h} I_{h, s} \\
& I_{h, a}^{\prime}=q v_{h} E_{h}-\gamma_{h} I_{h, a} \\
& R_{h}^{\prime}=\gamma_{h}\left(I_{h, s}+I_{h, a}\right) \\
& S_{v}^{\prime}=\mu_{v} N_{v}-b \beta_{h v} S_{v} \frac{I_{h, s}+I_{h, a}}{N_{h}}-\mu_{v} S_{v} \\
& E_{v}^{\prime}=b \beta_{h v} S_{v} \frac{I_{h, s}+I_{h, a}}{N_{h}}-\left(\mu_{v}+v_{v}\right) E_{v} \\
& I_{v}^{\prime}=v_{v} E_{v}-\mu_{v} I_{v} .
\end{aligned}
$$

\subsection{2 *Residence Times and Two-Patch Models}

The role of mobility between two communities, within the same city, living under dramatically distinct health, economic, social, and security settings is explored using a model as simple as possible, that is, a model that only considers two patches (prior modeling efforts that didn't account for the effective population size but that incorporated specific controls include [32]). Patch 2 has access to working health facilities, low crime rate, adequate human and financial resources, and adequate public health policies, in place. Patch 1 lacks nearly everything and crime is high. The differences in risk are captured by postulating very different transmission rates. We study the dynamics of host mobility in highly distinct environments, with risk being captured by the transmission rate, $\hat{\beta}$. Hence, $\hat{\beta}_{1} \gg \hat{\beta}_{2}$, where $\hat{\beta}_{i}$ defines the risk in Patch $i, i=1,2$ [Patch 1 (high risk) and Patch 2 (low risk)].

The host populations are stratified by epidemiological classes indexed by the patch of residence. In particular, $S_{h, i}, E_{h, i}, I_{h, a, i}, I_{h, s, i}$, and $R_{h, i}$ denote the susceptible, latent, infectious asymptomatic, infectious symptomatic, and recovered host populations in Patch $i, i=1,2$ with $S_{v, i}, E_{v, i}$, and $I_{v, i}$ denoting the susceptible, latent, and infectious mosquito populations in Patch $i, i=1,2$. As before, $N_{h, i}$ denotes the host patch population size $(i, i=1,2)$ and $N_{v, i}$ the total vector population in Patch $i, i=1,2$. The vector is assumed to be incapable of moving between patches, a reasonable assumption in the case of Aedes aegypti under the appropriate spatial scale. The patch model parameters are presented in Table 15.2 with the flow diagram, single patch dynamics model, capturing the situation when residents and visitors do not move; that is, when the $2 \times 2$ residence times matrix $\mathbb{P}$ is such that $p_{11}=p_{22}=1$ (Fig 15.10). 
Table 15.2 Description of the parameters used in system (15.6)

\begin{tabular}{l|l|l}
\hline Parameters & Description & Value \\
\hline$\beta_{v h}$ & Infectiousness of human to mosquitoes & 0.41 \\
\hline$\beta_{h v}$ & Infectiousness of mosquitoes to humans & 0.5 \\
\hline$b_{i}$ & Biting rate in Patch $i$ & 0.8 \\
\hline$v_{h}$ & Human'' incubation rate & $\frac{1}{7}$ \\
\hline$q$ & Fraction of latent that become asymptomatic and infectious & 0.1218 \\
\hline$\gamma_{i}$ & Recovery rate in Patch $i$ & $\frac{1}{5}$ \\
\hline$p_{i j}$ & Proportion of time residents of Patch $i$ spend in Patch $j$ & {$[0,1]$} \\
\hline$\mu_{v}$ & Vectors' natural mortality rate & $\frac{1}{13}$ \\
\hline$v_{v}$ & Vectors' incubation rate & $\frac{1}{9.5}$ \\
\hline
\end{tabular}



Fig. 15.10 Flow diagram of model (15.6)

Since individuals experience a higher risk of ZIKV infection while in Patch 1, then it is assumed that mobility from Patch 2 to Patch 1 is unappealing with typical Patch 2 residents spending (on the average) a reduced amount of time each unit of 
time in Patch 1. Parameters are chosen so that the dynamics of ZIKV within Patch 2 cannot be supported in the absence of mobility between Patch 1 and Patch 2. Thus, the Patch 2 local basic reproduction number is taken to be less than one, namely $\mathscr{R}_{02}=0.9$. Mobility is modeled under the residence times matrix $\mathbb{P}$ with entries given initially by $p_{21}=0.10$ and $p_{12}=0$.

Two cases are explored: A "worst case" scenario where control measures are hardly implemented due to crime, conflict, or other factors on Patch 1, that is, Patch 1 is a place where the risk of acquiring a ZIKV infection is high since $\mathscr{R}_{01}=2$. The "best case" scenario corresponds to the case when Patch 1 can implement some control measures with some degree of effectiveness, and consequently Patch 1 has an $\mathscr{R}_{01}=1.52$. The $\mathscr{R}_{0 i}$ values used are in line with those previously estimated for ZIKV outbreaks [31, 45]. Simulations are seeded by introducing an asymptomatic infected individual in Patch 1 under the assumption that the host and vector populations are fully susceptible in both patches.

Figure 15.11 (top) shows the incidence and final ZIKV epidemic size when Patch 1 is under the "worst case scenario," defined by a basic reproduction number of
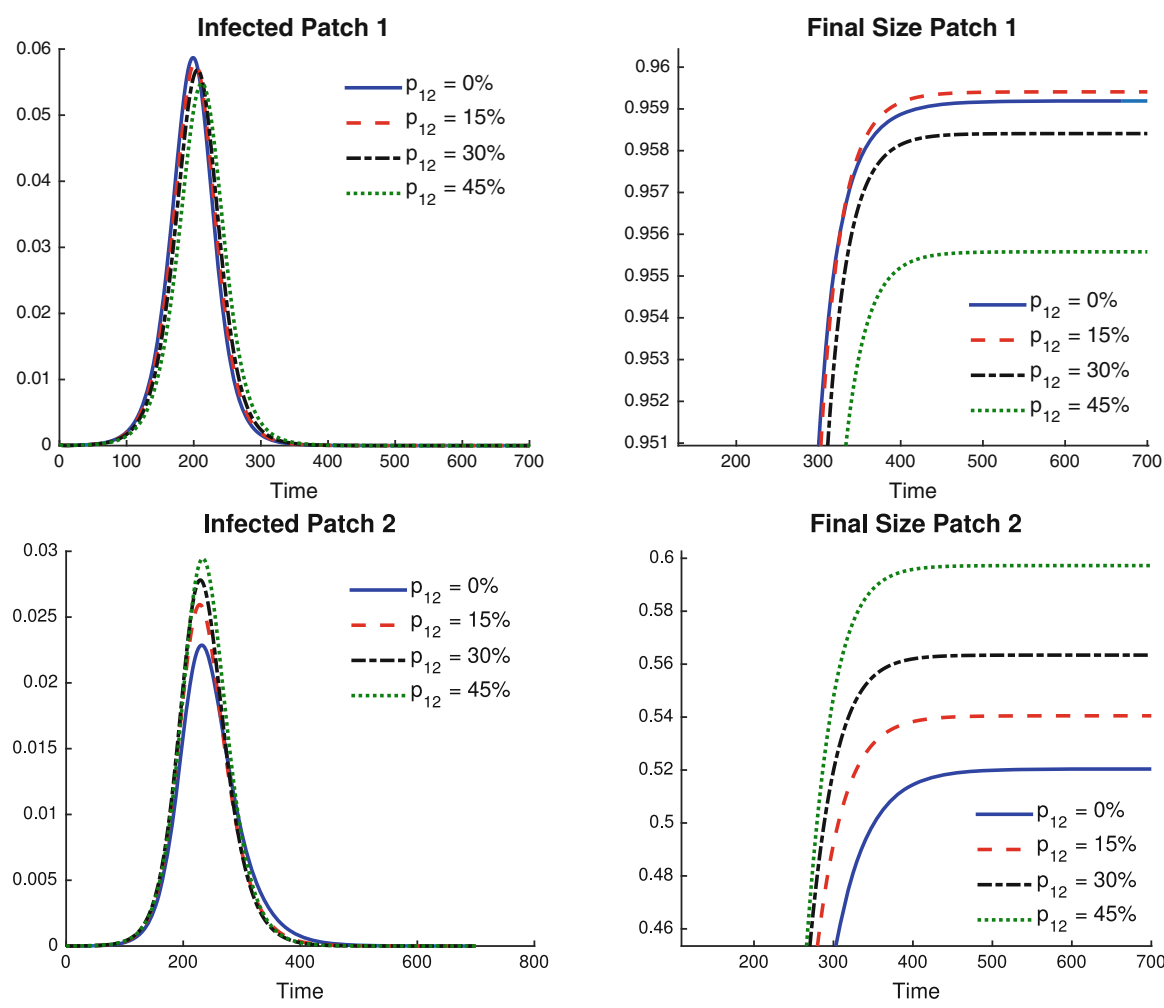

Final Size Patch 2

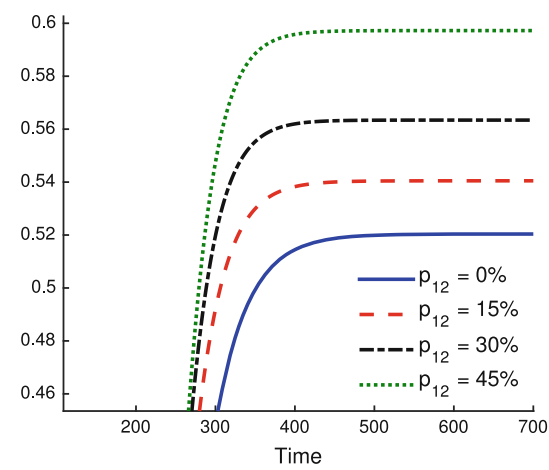

Fig. 15.11 Per patch incidence and final size proportions for $p_{21}=0.10, p_{12}=0,0.15,0.30$, and 0.45 . Mobility shifts the behavior of the Patch 1 final size in the "worst case" scenario: $\mathscr{R}_{01}=2$ and $\mathscr{R}_{02}=0.9$ 
$\mathscr{R}_{01}=2$ [45]. Figure 15.11 shows that at $p_{12}=0.15$ the final number of infected residents in Patch 1 is larger to the number in the baseline scenario $\left(p_{12}=0\right)$. In fact, it reaches almost $96 \%$ of the population, an unrealistic value. Additional simulated $p_{12}$ values show that final Patch 1 size would go below the baseline case; a benefit of mobility. Figure 15.11 highlights the case when the Patch 2 epidemic final size grows with increases in mobility when compared with the baseline case (no mobility from Patch 1). We see reductions in the Patch 1 epidemic final size for some mobility values accompanied by increments in the Patch 2 epidemic final size when compared to the baseline scenario (no mobility from Patch 1). Specifically, reductions in Patch 1 epidemic final size are around $1 \times 10^{-3}$, while increments in Patch 2 are around $1 \times 10^{-2}$, under the assumption that the population in Patch 1 is the same as that in Patch 2. Thus while mobility may provide benefits within Patch 1 (under the above assumptions) the fact remains that it does it at a cost. In short, it is also observed that the epidemic final size per patch does not respond linearly to changes in mobility even when only the mobility $p_{12}$ is increased (see Figs. 15.11 and 15.12).
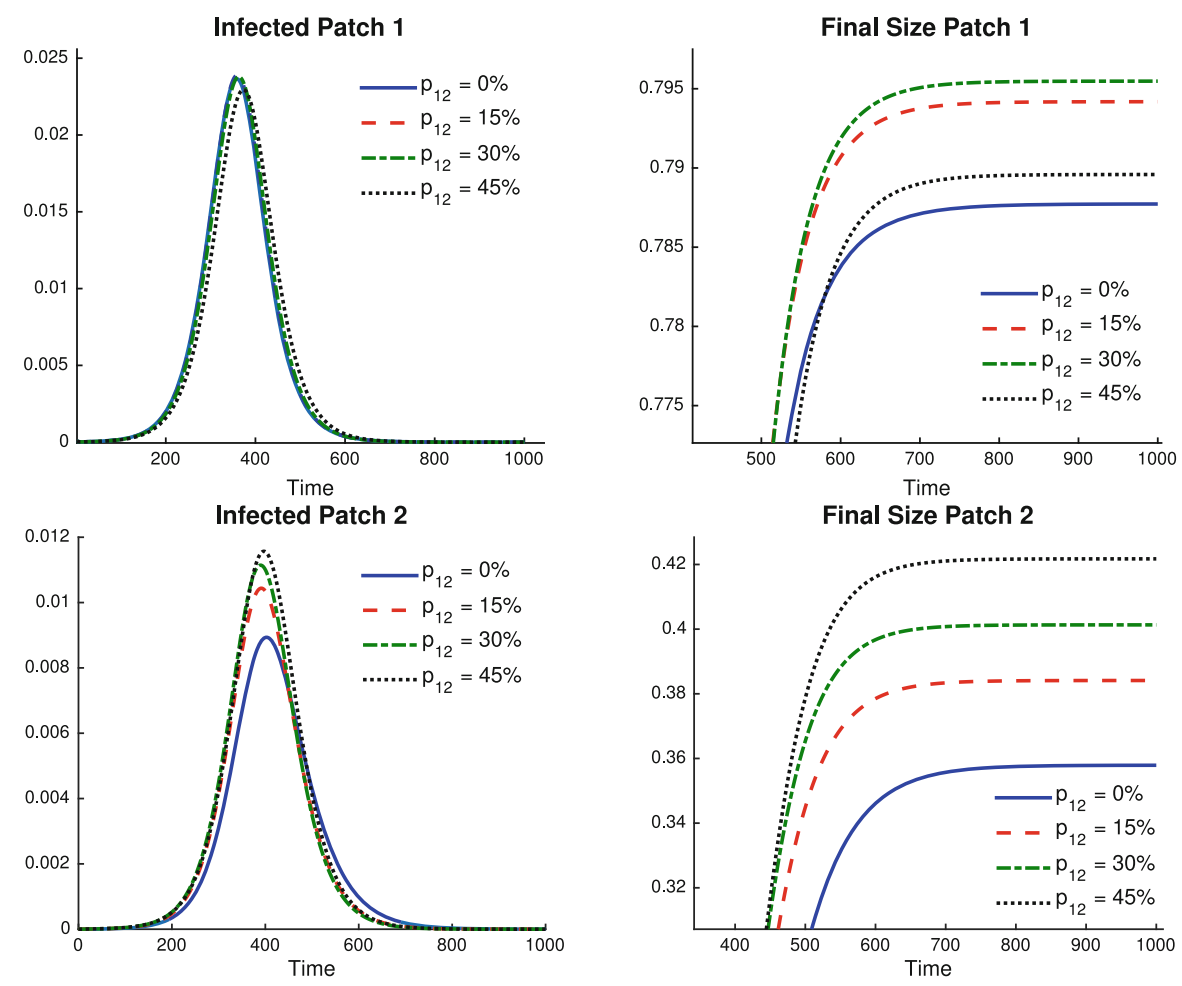

Fig. 15.12 Per patch incidence and final size proportions for $p_{21}=0.10, p_{12}=0,0.15,0.30$, and 0.45 . Mobility significantly shapes the per patch final sizes in the "worst case" scenario $\mathscr{R}_{01}=2$ and $\mathscr{R}_{02}=0.9$ 
Consider now the "best case" scenario, a basic reproduction number $\mathscr{R}_{01}=1.52$, under the assumption that the population in Patch 1 is the same as that in Patch 2. The results of simulations collected in Fig. 15.12 show a final size epidemic curve similar to that generated in the "worst case" scenario for Patch 1. Some mobility values can increase the Patch 1 epidemic final size, reaching almost $80 \%$ of the population when $p_{12}=0.30$, an unrealistic level, albeit, as expected lower than in the "worst" case scenario. The existence of a mobility threshold after which the final epidemic sizes in Patch 1 start to decrease is also observed. The results in Fig. 15.12 suggest that under all $p_{12}$ mobility levels, Patch 2 ZIKV epidemic final size supports monotone growth in the total number of infected individuals. The changes in the epidemic final size in each patch in Fig. 15.12 are roughly equivalent the same order, $1 \times 10^{-2}$ ) given that the population in Patch 1 is the same as that in Patch 2 .

The simulation results presented so far provide only partial information on the impact that short-term mobility may have on the transmission dynamics of ZIKV. Now, by fixing the mobility from Patch 2 to Patch 1, we are just focusing only on the impact of changes in mobility from Patch 1 to Patch 2. Further, the potential changes in mobility patterns that host populations may have in response to ZIKV dynamics are ignored by our use of a mobility matrix $\mathbb{P}$ with constant entries $p_{i j}$. We found that epidemic final size within Patch 1 is qualitatively similar in the worst and best case scenarios: increasing at first, decreasing after a certain threshold, and crossing down the baseline case under some mobility regimes. Further, it has been observed that the qualitative behavior of the epidemic final size in Patch 2 grows monotonically as mobility increases. Patch 1 and Patch 2 responses are of different orders of magnitude in the "worst case" scenario but roughly of the same order of magnitude in the "best case" scenario, which means, under our restrictive conditions and assumptions, that reductions in risk in Patch 1 do help significantly.

\subsubsection{1 *The Role of Risk Heterogeneity in the Dynamics of ZIKV Transmission}

The impact of risk heterogeneity on ZIKV dynamics within the overall twopatch system is explored, an analysis that requires the numerical estimation of the global reproduction number as a function of the mobility matrix $\mathbb{P}$. Using the previous scenarios $\left(\mathscr{R}_{01}=1.52,2\right)$ simulations are carried out first assuming equal population sizes $\left(N_{1}=N_{2}\right)$. However, when looking at the impact of changes in risk on Patch $2\left(\mathscr{R}_{02}=0.8,0.9,1,1.1\right)$, our simulations identify a growing epidemic in Patch 2 as risk increases with the overall community experiencing nonlinear changes in risk as residency times change from the baseline scenario given by $p_{12}=0$. Specifically, Fig. 15.13 captures overall reductions on the global reproduction number for all residence times while identifying the existence of a residence time interval for which mobility decreases the total size of the outbreak in the two-patch community, when compared to the corresponding baseline case $\left(p_{12}=0\right)$. In the absence of mobility from Patch $1\left(p_{12}=0\right)$, increases in the epidemic final size as $\mathscr{R}_{0 i}$ increases are observed. These simulations show that 

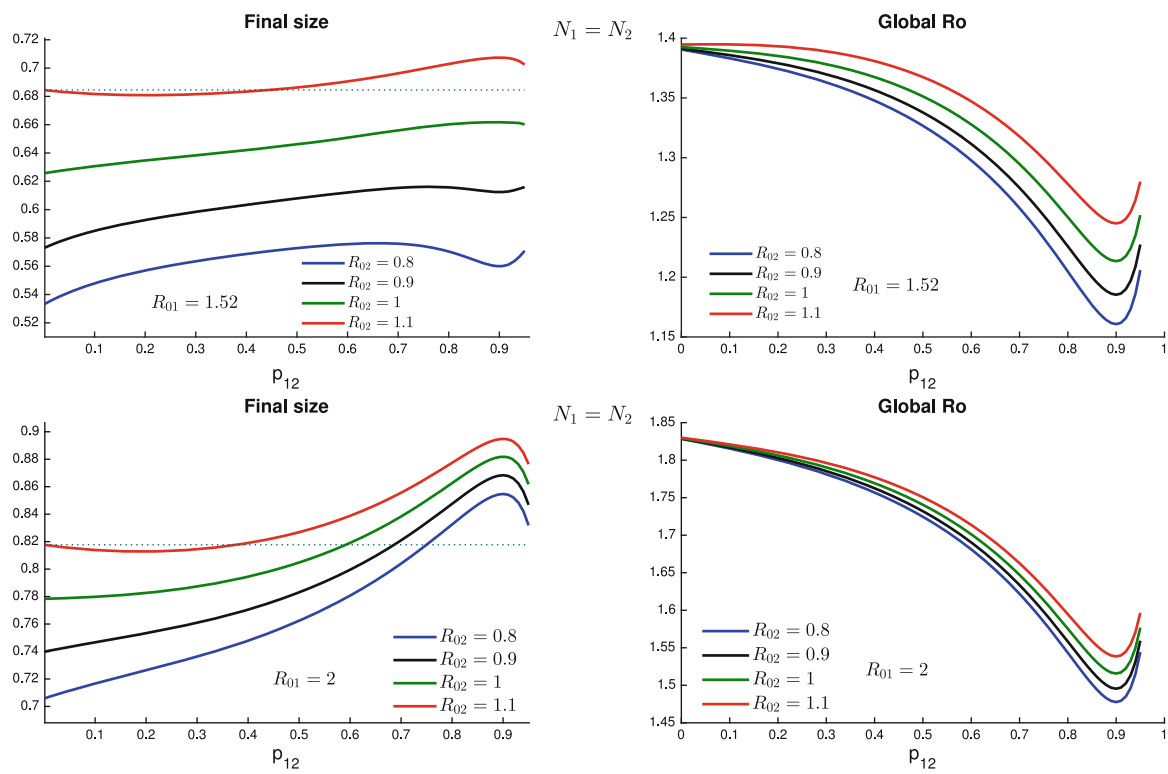

Fig. 15.13 Local and global final sizes through mobility values when $p_{21}=0.10$. Although mobility reduces the global $\mathscr{R}_{0}$, allowing mobility in the case of El Salvador $\left(\mathscr{R}_{0}=2\right)$ might lead to a detrimental effect in the global final size

mobility can slow down the speed of the outbreak (smaller global $\mathscr{R}_{0}$ ). Of course, the simulation results also re-affirm the obvious, that is, that the existence of a high risk, mobile, and well-connected patch can serve as an outbreak magnifier; a situation that has been explored within an $n$-patch system under various connective schemes $[7,10]$. This is because, in the two-patch case, it is observed that the global reproduction number $\mathscr{R}_{0}$ experiences reductions for almost all mobility values. For the scenarios selected $\mathscr{R}_{0}$ never drops below 1 . Hence, under our assumptions and scenarios, it is seen that the use of fixed mobility patterns makes the elimination of ZIKV extremely difficult if not impossible under our two scenarios. Figure 15.13 provides an example that highlights the relationship between the global reproduction number and corresponding epidemic final size.

\subsubsection{2 *The Role of Population Size Heterogeneity in the Dynamics of ZIKV Transmission}

The role of population density in the total epidemic final size and global basic reproduction number is explored under our two scenarios, now under the changed assumption that the densities (population sizes) of Patch 1 and Patch 2 differ. Specifically, we take $N_{1}=2 N_{2}, 3 N_{2}, 5 N_{2}$, and $10 N_{2}$. 

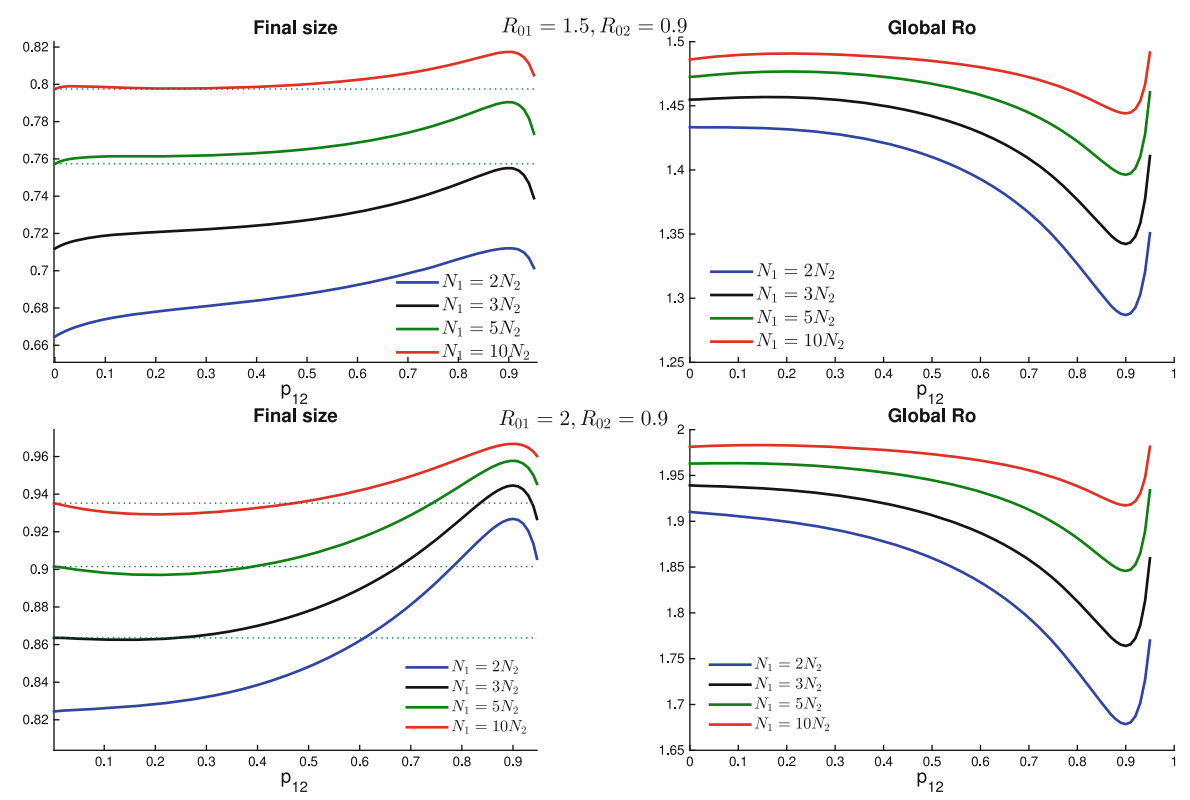

Fig. 15.14 Total final size and global basic reproduction number through mobility values when $p_{21}=0.10$. Local risk values are set up to $\mathscr{R}_{02}=0.9$ and $\mathscr{R}_{01}=1.52,2$

It is observed that difference in population sizes do matter. Specifically, it is observed that (under our selections) a big difference in density indicate that a higher epidemic final size is reached. The value of $90 \%$ for the "worst case" is possible with changes in the global reproduction number exhibiting different patterns (see Fig. 15.14). We observe that despite increases in the total epidemic final size as mobility changes the global $\mathscr{R}_{0}$ actually decreases monotonically for most residence times, never falling below one. A sensible degree of magnification on the spread of the disease as residence times change is observed whenever the differences between $N_{1}$ and $N_{2}$ are not too extreme. In fact, it is possible for mobility to be beneficial in the control of ZIKV under the above simplistic extreme scenarios. Simulations continue to show that under the prescribed conditions and assumptions, model generated ZIKV outbreaks remain unrealistically high. The simulations show, for example, that the global reproduction number reaches its minimum at around $p_{12}=0.90$ with Fig. 15.14 showing that the larger the high risk population gets $\left(N_{1}>>N_{2}\right)$, the greater the total epidemic final size becomes as individuals from Patch 1 spend more than half of their day in Patch 2 . Using a low $p_{12}$ value, a small benefit is observed, namely the total epidemic final size is reduced, when the differences between $\mathscr{R}_{0 i}$ are high.

For the two epidemiological scenarios $\mathscr{R}_{01}=2$ and $\mathscr{R}_{01}=1.52$, Tables 15.3 and 15.4 provide a summary of the average proportion of infected for low 
Table 15.3 Final size (Patch 1, Patch 2) $N_{1}=10,000, \mathscr{R}_{01}=2, \mathscr{R}_{02}=0.9$, and $p_{21}=0.10$

\begin{tabular}{l|l|l|l|l}
\hline$N_{2}$ & Low mobility & Intermediate mobility & High mobility & Min $\mathscr{R}_{0}$ \\
\hline$N_{1}=N_{2}$ & $(0.9594,0.5333)$ & $(0.9583,0.5633)$ & $(0.9539,0.6122)$ & 1.4954 \\
\hline$N_{1}=2 N_{2}$ & $(0.9683,0.5418)$ & $(0.9685,0.5599)$ & $(0.9667,0.6116)$ & 1.6786 \\
\hline$N_{1}=3 N_{2}$ & $(0.9709,0.5390)$ & $(0.9713,0.5478)$ & $(0.9701,0.6018)$ & 1.7640 \\
\hline$N_{1}=5 N_{2}$ & $(0.9729,0.5283)$ & $(0.9732,0.5255)$ & $(0.9725,0.5852)$ & 1.8457 \\
\hline$N_{1}=10 N_{2}$ & $(0.9741,0.5030)$ & $(0.9743,0.4908)$ & $(0.9739,0.5624)$ & 1,9173 \\
\hline
\end{tabular}

Table 15.4 Final size (Patch 1, Patch 2) $N_{1}=10,000, \mathscr{R}_{01}=1.52, \mathscr{R}_{02}=0.9$, and $p_{21}=0.10$

\begin{tabular}{l|l|l|l|l}
\hline$N_{2}$ & Low mobility & Intermediate mobility & High mobility & Min $\mathscr{R}_{0}$ \\
\hline$N_{1}=N_{2}$ & $(0.7920,0.3756)$ & $(0.7950,0.4010)$ & $(0.7849,0.4304)$ & 1.1853 \\
\hline$N_{1}=2 N_{2}$ & $(0.8287,0.3938)$ & $(0.8340,0.4061)$ & $(0.8300,0.4356)$ & 1.3023 \\
\hline$N_{1}=3 N_{2}$ & $(0.8398,0.3948)$ & $(0.8448,0.3956)$ & $(0.8422,0.4248)$ & 1.3590 \\
\hline$N_{1}=5 N_{2}$ & $(0.8480,0.3877)$ & $(0.8520,0.3731)$ & $(0.8500,0.4046)$ & 1.4141 \\
\hline$N_{1}=10 N_{2}$ & $(0.8533,0.3652)$ & $(0.8556,0.3352)$ & $(0.8542,0.3756)$ & 1.4630 \\
\hline
\end{tabular}


Fig. 15.15 Global $\mathscr{R}_{0}$ dynamics through mobility when $p_{21}=0.10$. Patch 2 populations vary from $N_{1}=N_{2}, 2 N_{2}, 3 N_{2}, 5 N_{2}$ up to $N_{1}=10 N_{2}$. The global $\mathscr{R}_{0}$ hits its minimum always at an unrealistic $91 \%$ of mobility. As $N_{1}$ approaches $N_{2}$, this minimum value decreases

$\left(p_{12}=0-0.2\right)$, intermediate $\left(p_{12}=0.2-0.4\right)$, and high mobility $\left(p_{12}=0.4-0.6\right)$ when $p_{21}=0.10$. The role of population scaling $N_{1}=2 N_{2}, 3 N_{2}, 5 N_{2}$, and $10 N_{2}$ is also explored. Figure 15.15 shows the global $\mathscr{R}_{0}$ over all mobility values for different population weights in the two epidemic scenarios. The minimum $\mathscr{R}_{0}$ value is reached for all cases when mobility is at an unrealistic $91 \%$ and when $N_{1} \approx N_{2}$. The results collected in Fig. 15.15 show that short-term mobility plays an important role in ZIKV dynamics, again, under a system involving two highly differentiated patches. Simulations also suggest that, even though mobility can reduce the global reproduction number, mobility by itself is not enough to eliminate an outbreak or make a real difference under our two scenarios. 


\subsubsection{What Did We Learn from These Single Outbreak Simulations?}

The study of the role of mobility at large spatial scales may be best captured using question-specific related models that account for the possibility of long-term mobility (see, for example, [2, 3, 18, 22, 27, 28, 30, 43]). Here, we made use of two patches, as distinct as they can be would be able to shed some light on the transmission dynamics of ZIKV, whenever extreme health disparities within neighboring communities or within urban centers were the norm. Although the goal is not to fit specific outbreaks, we decided to make use of recently published parameter ranges, including some reported by us [45]. The impact of ZIKV can be assessed locally (each patch) or globally, that is, over the two-patch system. Here, system risk assessment was carried out by computing $\mathscr{R}_{0}$, via the numerical solution of a system of nonlinear equations. Changes in the system $\mathscr{R}_{0}$ were computed (as residence times were varied) in relationship to the local $\mathscr{R}_{0 i}$, that is, local basic reproduction numbers (in the absence of mobility). Further, the mobility-dependent system epidemic final sizes were computed via simulations that assessed the impact of mobility (and risk) locally and on the overall system. The metrics used in our assessment included the overall epidemic final size (a measure of the overall impact of an outbreak), a function of mobility within the two selected scenarios $\left(\mathscr{R}_{01}=1.52\right.$ and $\left.\mathscr{R}_{01}=2\right)$.

The challenges posed by policies that may be beneficial to the system but detrimental to each patch were explored within our two-patch system. Situations where the total final epidemic size increased with increments in $\mathscr{R}_{02}$, and situations where the total final epidemic size decreased under low mobility values for $\mathscr{R}_{(02)}$ were documented. Population density does make a difference and examples when $\mathscr{R}_{02}<1$ with mobility incapable of reducing the total epidemic final size under no differences in patch density (here measured by total population size in each patch, both assumed to have roughly the same area) were also identified. Differences in population density were also shown to be capable of generating reductions on the total final epidemic size within some mobility regimes.

The highly simplified two-patch model used seemed to have shed some light on the role of mobility on the spread of ZIKV in areas where huge differences in the availability of public health programs and services - the result of endemic crime, generalized violence, and neglect - exist. Model simulations seemed to have shed some light on the potential relevance of factors that we failed to account for. The value of the use of single patch-specific risk parameters $(\hat{\beta})$ has strengths and limitations. The model used did not account explicitly for changes in the levels of infection within the vector population nor did it account for the impact of substantial differences in patch vector population sizes. The simplified model failed to account for the responses to outbreaks by patch residents as individuals may alter mobility patterns, use more protective clothing while responding individually and independently to official control programs in the face of dramatic increases on the vector population or due to a surge in the number of cases. The use of two patches 
and severe assumptions limits the outcomes that such an oversimplified system can support. Communities can't in generally be modeled under a highly differentiated two-tier system and in the case of ZIKV, the possibility of vertical transmission in humans and vectors as well as sexually transmitted ZIKV cannot be completely neglected $[8,39]$. The introduction of changes in behavior in response to individuals' assessment of the levels of risk infection over time needs to be addressed [10]; a challenge that has yet to be met to the satisfaction of the scientific community involved in the study of epidemiological processes as complex adaptive systems (see, for example, [26, 36, 42]).

The limitations of the role of technology in the absence of the public health infrastructure-there is no silver bullet-have been addressed in the context of Ebola [16, 49]. It would be interesting to see the impact of technology in settings where health disparities are pervasive, using a two-patch Lagrangian epidemic model in the context of communicable and vector-borne diseases, including dengue, tuberculosis , and Ebola [5, 23, 34, 35]. Further, its often the case that the use of simplified models quite often overestimates the impact of an outbreak (see $[37,38]$ ) and so find the right level of model heterogeneity (number of patches) becomes a pressing and challenging question. What is the right level of aggregation to address these questions?

Certainly, we have seen the use of dramatic measures to limit the spread of diseases like SARS, influenza , or Ebola [16, 17, 27], as well as the rise of vectorborne diseases like dengue and Zika, and the dramatic implications that some measures have had on local and global economies. The question remains, what can we do to mitigate or limit the spread of disease, particularly emergent diseases without disrupting central components? Discussions on these issues are recurrent $[26,36]$, most intensely in the context of SARS, influenza, Ebola , and Zika, in the last decade or so. The vulnerability of world societies is directly linked to the lack of action in addressing the challenges faced by the weakest links in the system. This must be accepted and acted on by the world community. We need global investments in communities and nations where health disparities and lack of resources are the norm. We must invest in research and surveillance within clearly identified world hot spots, where the emergence of new diseases is most likely to occur. We must do so with involvement at all levels of the affected communities [12, 44].

\section{References}

1. Andrews, J.R., C. Morrow, and R. Wood (2013) Modeling the role of public transportation in sustaining tuberculosis transmission in South Africa. Am J Epidemiol 177: 556-561.

2. Banks, H.T. and C. Castillo-Chavez (2003)Bioterrorism: Mathematical Modeling Applications in Homeland Security. SIAM, 2003.

3. Baroyan, O., L. Rvachev, U. Basilevsky, V. Ermakov, K. Frank, M. Rvachev, and V. Shashkov (1971) Computer modelling of influenza epidemics for the whole country (USSR), Advances in Applied Probability 3: 224-226. 
4. Bichara, D. and C. Castillo-Chavez (2016) Vector-borne diseases models with residence timesa Lagrangian perspective, Math. Biosc. 281: 128-138.

5. Bichara, D., S. A. Holechek, J. Velázquez-Castro, A. L. Murillo, and C. Castillo-Chavez (2016) On the dynamics of dengue virus type 2 with residence times and vertical transmission. Letters in Biomathematics 3:140-160.

6. Bichara, D. and A. Iggidr (2017) Multi-patch and multi-group epidemic models: a new framework. J. Math. Biol. https://doi.org/10.1007/s285-017-1191-9.

7. Bichara, D., Y. Kang, C. Castillo-Chavez, R. Horan, and C. Perrings (2015) SIS and SIR epidemic models under virtual dispersal, Bull. Math. Biol. 77: 2004-2034.

8. Brauer, F., C. Castillo-Chavez, A. Mubayi, and S. Towers (2016) Some models for epidemics of vector-transmitted diseases. Infectious Disease Modelling 1: 79-87.

9. Brauer,F. and C. Castillo-Chavez (2012) Mathematical Models for Communicable Diseases 84 SIAM.

10. Castillo-Chavez, C., K. Barley, D. Bichara, D. Chowell, E.D. Herrera, B. Espinoza, V. Moreno, S. Towers, and K. Yong (2016) Modeling Ebola at the mathematical and theoretical biology institute (MTBI). Notices of the AMS 63.

11. Castillo-Chavez, C., D. Bichara, and B.R. Morin (2016) Perspectives on the role of mobility, behavior, and time scales in the spread of diseases, Proc. Nat. Acad. Sci. 113: 14582-14588.

12. Castillo-Chavez, C., R. Curtiss, P. Daszak, S.A. Levin, O. Patterson-Lomba, C. Perrings, G. Poste, and S. Towers (2015) Beyond Ebola: Lessons to mitigate future pandemics. The Lancet Global Health 3: e354-e355.

13. Castillo-Chavez, C., B. Song, and J. Zhangi (2003) An epidemic model with virtual mass transportation: The case of smallpox. Bioterrorism: Mathematical Modeling Applications in Homeland Security 28: 173.

14. CDC(a) (2016) Zika virus. Online, February 01, 2016.

15. Chatterjee, D. and A. K. Pramanik (2015) Tuberculosis in the African continent: A comprehensive review. Pathophysiology 22: 73-83.

16. Chowell, D., C. Castillo-Chavez, S. Krishna, X. Qiu, and K.S. Anderson (2015) Modelling the effect of early detection of Ebola. The Lancet Infectious Diseases

17. Chowell, G., P.W. Fenimore, M.A. Castillo-Garsow, and C. Castillo-Chavez (2003) SARS outbreaks in Ontario, Hong Kong and Singapore: the role of diagnosis and isolation as a control mechanism, J. Theor. Biol. 224: 1-8.

18. Chowell, G., J.M. Hyman, S. Eubank, and C. Castillo-Chavez (2003) Scaling laws for the movement of people between locations in a large city. Phys. Rev. 68: 066102.

19. Daniel, T.M. (2006) The history of tuberculosis. Respiratory Medicine 100: 1862-1870.

20. de Oliveira, G.P., A.W. Torrens, P. Bartholomay, and D. Barreira (2013) Tuberculosis in Brazil: last ten years analysis - 2001-2010 in Journal of Infectious Diseases 17: 218-233.

21. Duffy, M.R., T.-H. Chen, W.T. Hancock, A.M. Powers, J.L. Kool, R.S. Lanciotti, M. Pretrick, M. Marfel, S. Holzbauer, C. Dubray, et al (2009) Zika virus outbreak on yap island, federated states of Micronesia. New England J. Medicine 360: 2536-2543.

22. Elveback, L.R., J.P. Fox, E. Ackerman, A. Langworthy, M. Boyd, and L. Gatewood (1976) An influenza simulation model for immunization studies. Am. J. Epidemiology 103: 152-165.

23. Espinoza, B., V. Moreno, D. Bichara, and C. Castillo-Chavez (2016) Assessing the efficiency of movement restriction as a control strategy of Ebola. In Mathematical and Statistical Modeling for Emerging and Re-emerging Infectious Diseases, pages 123-145. Springer.

24. Eubank, S., H. Guclu, V. A. Kumar, M.V. Marathe, A. Srinivasan, Z. Toroczkai, and N. Wang (2004) Modelling disease outbreaks in realistic urban social networks. Nature 429: 180-184.

25. Feng, Z., C. Castillo-Chavez, and A.F. Capurro (2000)A model for tuberculosis with exogenous reinfection. Theor. Pop. Biol. 57: 235-247.

26. Fenichel, E.P., C. Castillo-Chavez, M.G. Ceddia, G. Chowell, P.A.G. Parra, G.J. Hickling, G. Holloway, R. Horan, B. Morin, C. Perrings, et al (2011) Adaptive human behavior in epidemiological models. Proc. Nat. Acad. Sci. 108: 6306-6311. 
27. Herrera-Valdez, M.A., M. Cruz-Aponte, and C. Castillo-Chavez (2011) Multiple outbreaks for the same pandemic: Local transportation and social distancing explain the different "waves" of A-H1N1pdm cases observed in México during 2009. Math. Biosc. Eng. 8: 21-48.

28. Hyman, J.M. and T. LaForce (2003) Modeling the spread of influenza among cities. In Bioterrorism: Mathematical Modeling Applications in Homeland Security, pages 211-236. SIAM.

29. Jaitman, L. (2015) Los costos del crimen y la violencia en el bienestar en America Latina y el Caribe, L. Jaitman Ed. Banco Interamericano del Desarrollo.

30. Khan, K., J. Arino, W. Hu, P. Raposo, J. Sears, F. Calderon, C. Heidebrecht, M. Macdonald, J. Liauw, A. Chan, et al (2009) Spread of a novel influenza a (H1N1) virus via global airline transportation. New England J. Medicine 361: 212-214.

31. Kucharski, A.J., S. Funk, R.M. Eggo, H.-P. Mallet, J. Edmunds, and E.J. Nilles (2016) Transmission dynamics of Zika virus in island populations: a modelling analysis of the 201314 French Polynesia outbreak. bioRxiv, page 038588.

32. Lee, S. and C. Castillo-Chavez (2015) The role of residence times in two-patch dengue transmission dynamics and optimal strategies, J. Theor. Biol. 374:152-164.

33. Luzze, H., D.F. Johnson, K. Dickman, H. Mayanja-Kizza, A. Okwera, K. Eisenach, M. D. Cave, C.C. Whalen, J.L. Johnson, W.H. Boom, M. Joloba, and Tuberculosis Research Unit (2013) Relapse more common than reinfection in recurrent tuberculosis 1-2 years post treatment in urban Uganda. Int. J. of Tuberculosis and Lung Disease 17: 361-367.

34. Moreno, V., B. Espinoza, K. Barley, M. Paredes, D. Bichara, A. Mubayi, and C. CastilloChavez (2017) The role of mobility and health disparities on the transmission dynamics of tuberculosis. Theoretical Biology and Medical Modelling 14 :3, 2017.

35. Moreno, V.M., B. Espinoza, D. Bichara, S. A. Holechek, and C. Castillo-Chavez (2017) Role of short-term dispersal on the dynamics of Zika virus in an extreme idealized environment. Infectious Disease Modelling 2: 21-34.

36. Morin, B.R., E.P. Fenichel, and C. Castillo-Chavez (2013) SIR dynamics with economically driven contact rates, Natural resource modeling 26: 505-525.

37. Nishiura, H., C. Castillo-Chavez, M. Safan, and G. Chowell (2009) Transmission potential of the new influenza A (H1N1) virus and its age-specificity in Japan, Eurosurveillance 14: 19227.

38. Nishiura, H., G. Chowell, and C. Castillo-Chavez (2011) Did modeling overestimate the transmission potential of pandemic (H1N1-2009)? sample size estimation for post-epidemic seroepidemiological studies. PLoS One 6: e17908.

39. Padmanabhan, P., P. Seshaiyer, and C. Castillo-Chavez (2017) Mathematical modeling, analysis and simulation of the spread of Zika with influence of sexual transmission and preventive measures. Letters in Biomathematics 4: 148-166.

40. Patterson-Lomba, O., E. Goldstein, A. Gómez-Liévano, C. Castillo-Chavez, and S. Towers (2015)Infections increases systematically with urban population size: a cross-sectional study. Sex Transm Infect 91: 610-614.

41. Patterson-Lomba, O., M. Safan, S. Towers, and J. Taylor (2016) Modeling the role of healthcare access inequalities in epidemic outcomes. Math. Biosc. Eng. 13: 1011-1041.

42. Perrings, C. Castillo-Chavez, G. Chowell, P. Daszak, E.P. Fenichel, D. Finnoff, R.D. Horan, A.M. Kilpatrick, A.P. Kinzig, N.V. Kuminoff, et al (2014) Merging economics and epidemiology to improve the prediction and management of infectious disease. EcoHealth 11: 464-475.

43. Rvachev, L.A. and I.M. Longini Jr. (1985) A mathematical model for the global spread of influenza. Math. Biosc. 75: 3-22.

44. Stroud, P., S. Del Valle, S. Sydoriak, J. Riese, and S. Mniszewski (2007) Spatial dynamics of pandemic influenza in a massive artificial society. Journal of Artificial Societies and Social Simulation 10: 9.

45. Towers, S., F. Brauer, C. Castillo-Chavez, A.K. Falconar, A. Mubayi, and C.M. Romero-Vivas (2016) Estimation of the reproduction number of the 2015 Zika virus outbreak in Barranquilla, Colombia, and estimation of the relative role of sexual transmission. Epidemics 17: 50-55. 
46. Towers, S., O. Patterson-Lomba, and C. Castillo-Chavez (2014) Temporal variations in the effective reproduction number of the 2014 West Africa Ebola outbreak. PLoS currents 6.

47. Verver, S., R.M. Warren, N. Beyers, M. Richardson, G.D. van der Spuy, M.W. Borgdorff, D. A. Enarson, M.A. Behr, and P.D. van Helden (2005) Rate of reinfection tuberculosis after successful treatment is higher than rate of new tuberculosis, Am. J. Respiratory and Critical Care Medicine 171: 1430-1435.

48. W. H. O. (WHO) (2015) Tuberculosis, fact sheet no. 104. Online, October 2015.

49. Yong, K., E.D. Herrera, and C. Castillo-Chavez (2016)From bee species aggregation to models of disease avoidance: The Ben-Hur effect. In Mathematical and Statistical Modeling for Emerging and Re-emerging Infectious Diseases, pages 169-185. Springer.

50. Zhao, H., Z. Feng, and C. Castillo-Chavez (2014) The dynamics of poverty and crime. J. Shanghai Normal University (Natural Sciences. Mathematics), pages 225-235. 